\title{
Abnormal excess heat observed during Mizuno-type experiments
}

\author{
Jean-François FAUVARQUE*, Pierre Paul CLAUZON, Gérard Jean-Michel LALLEVÉ \\ Laboratoire d'Electrochimie Industrielle, Conservatoire National des Arts et Métiers \\ 292, rue Saint Martin, F-75141 PARIS, CEDEX 03, France. \\ *e-mail : fauvarqu@cnam.fr
}

\begin{abstract}
A simple calorimeter has been designed that works at constant temperature; that of boiling water. Heat losses can be estimated accurately with an ohmic heater. As expected, losses are independent of the electric power input to the heater and the amount of evaporated water is linearly dependant on the power input. The device has been used to determine the heating power of a plasma electrolysis (the OhmoriMizuno experiment). We confirm that in this experiment, the heat output from electrolysis is greater than the electrical power input. The excess energy increases as the electrolysis voltage is increased from $200 \mathrm{~V}$ up to $350 \mathrm{~V}$ (400 W input). The excess energy may be as high as $120 \mathrm{~W}$.
\end{abstract}

\section{1- INTRODUCTION}

Our experiment is an electrolysis experiment made in plasma mode with a Tungsten cathode, a platinized Titanium wire as an anode, and as electrolyte a water solution of potassium carbonate ( $\mathrm{K} 2 \mathrm{CO} 3$ at $0.2 \mathrm{M})$. In the same type of experiments, T. Mizuno reported both excess heat and excess hydrogen production.

The explanations found in the bibliography for this phenomenon are, for example, transmutations in the cathode material (2). The abnormal observations are concerned with the appearance of Osmium, Iridium Platinum and Gold, without quantitative measurements. (2) 
We thought it would be quite interesting to confirm these results with the help of a simplified experimental setup.

\section{2- EXPERIMENTAL}

We decided to do our experiments at a constant temperature, the boiling point of water, and to measure the heat emissions only by weighing the mass of water in the experimental device. A water storage container inside the device allows us to maintain the electrolyte at a constant water level, and thus keep the $\mathrm{K}_{2} \mathrm{CO}_{3}$ concentration constant. This container, being graduated, also gives us a way to confirm the mass of water boiled off.

An interesting repercussion of this operating mode concerns the thermal losses. Once the boiling temperature is reached and overall thermal stability is achieved, the thermal losses, which depend only on the temperature, remain constant, and this is true whatever thermal emissions are observed. This allowed us to hold the experiment in a simple beaker of an appropriate size and then to avoid the of an opaque Dewar vessel. This allows us to visually observe the cathode condition.

Finally, the use of an ohmic heater allowed us to bring the device at the suitable boiling temperature and to maintain this temperature at this level much more quickly than by electrolysis alone. We also verified that other sources of inaccuracy were insignificant. For example, in the range of power used, the recondensation level inside the device was low, and few unboiled droplets of water sprayed outside the beaker. The excess heat that we were trying to measure is quite large (30\% to 100\%). So, it is not necessary to obtain very precise measurements. 


\section{3- EXPERIMENTAL DEVICE DESCRIPTION}

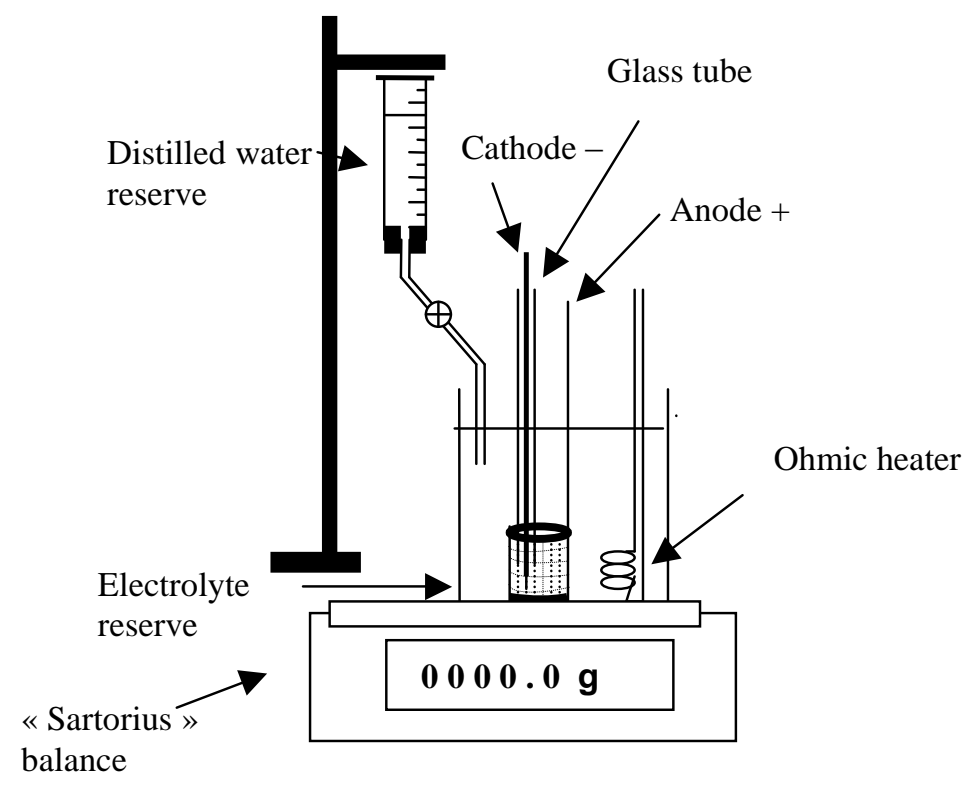

Figure 1. Experimental setup. Electrolyte reserve is $2 \mathrm{l}$. The tripod used to hold components in position is not shown

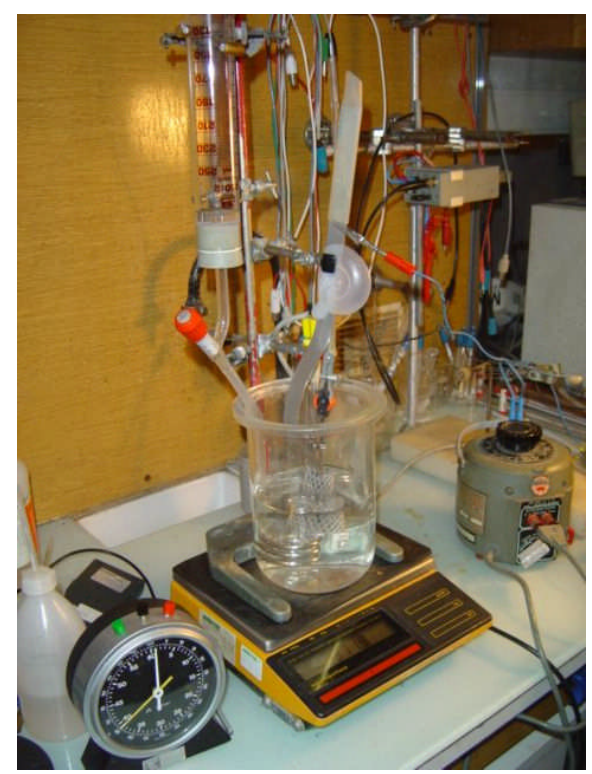

Figure 2. Photograph of the experimental apparatus. 
Figure 1 shows the details of the experimental setup:

- $\quad$ A SARTORIUS balance, the heart of the device, measuring up to $6 \mathrm{~kg}$ at an accuracy of $0.1 \mathrm{~g}$

- $\quad$ Beaker containing between 1 to 1.5 liter of electrolyte

- Tripod (not shown in this figure) is placed on the balance to hold the electrodes and a container of pure water

- Continuous current electricity supply (500 volts, 4 amperes)

- Wattmeter to measure the input energy (Unigor 390 LEM)

The input energy was also frequently measured by use of the inlet voltage and the mean current intensity as given by a recorder. These results were compared to the wattmeter data.

- $\quad$ A tungsten cathode of $2.4 \mathrm{~mm}$ diameter made with $2 \%$ of Thorium (this type of electrode is often used for commercial scale welding)

This cathode rod is shielded with a Pyrex tube. About $15 \mathrm{~mm}$ of the rod extends out from the tube, where it is exposed to electrolysis. In some experiments, ceramic tubes were used instead of Pyrex.

- An anode made of a wire in platinized titanium in a cone or cylinder shape around the cathode to regulate the water current. This is made quite large, to cope with the boiling, and to stop unboiled droplets of electrolyte from leaving the beaker. The anode and cathode are $4 \mathrm{~cm}$ apart.

- An ohmic heater rated to about 400 watts (enough to evaporate $34 \mathrm{~g}$ of water in 300 seconds)

- A distillated water container equipped with a tap which allows us to maintain a stable water level inside the beaker during the experiment.

- Various measuring instruments for the electrical components.

A typical run lasts 10 to 20 minutes, divided into 5-minute measurement periods. The evaporated water quantity may reach 50 to $100 \mathrm{~g}$, which corresponds to energy ranging from 113,000 to 226,000 joules. In some cases, we used a Geiger-Muller device to check for possible nuclear radiation. The results of these nuclear observations were always negative.

\section{4-1 CALIBRATION}

Using an ohmic heater, which can remain inside the beaker permanently, we have verified the response of our device to heat emission. It was necessary to verify that the thermal losses were constant beyond a certain input power level, and then that every increase in power would give a loss of water corresponding exactly to the energy supplied in the device during the measurement period.

The electrolyte level is maintained constant, due to the supply of water (at $20^{\circ} \mathrm{C}$ ) from the reserve. The energy used for the boiling of $1 \mathrm{~g}$ of water is then: 
$2260 \mathrm{j}$ (latent heat at $\left.100^{\circ} \mathrm{C}\right)+\left(100^{\circ} \mathrm{C}-20^{\circ} \mathrm{C}\right) \times 1 \times 4.18$ (heat needed to bring $1 \mathrm{~g}$ of water from $20^{\circ} \mathrm{C}$ to $\left.100^{\circ} \mathrm{C}\right)=2594 \mathrm{j}$.

Figure 3, below, shows the results. Note that from about 300 watts inlet power, the experimental curve and the theoretical straight line are rigorously parallel, and the thermal loss is 75 watts. Three important features can be deduced when we maintain the power of the ohmic heater at about 400 watts:

- We were able to verify the validity of our measurement of the latent heat of water evaporation

- The power increase due to the experiment is directly measured by the water loss during a given time unit.

- Losses due to the recondensation inside the experimental device, as well as those caused by unboiled droplets driven out of the beaker, seem to be negligible.

Vaporized water with re-feeding

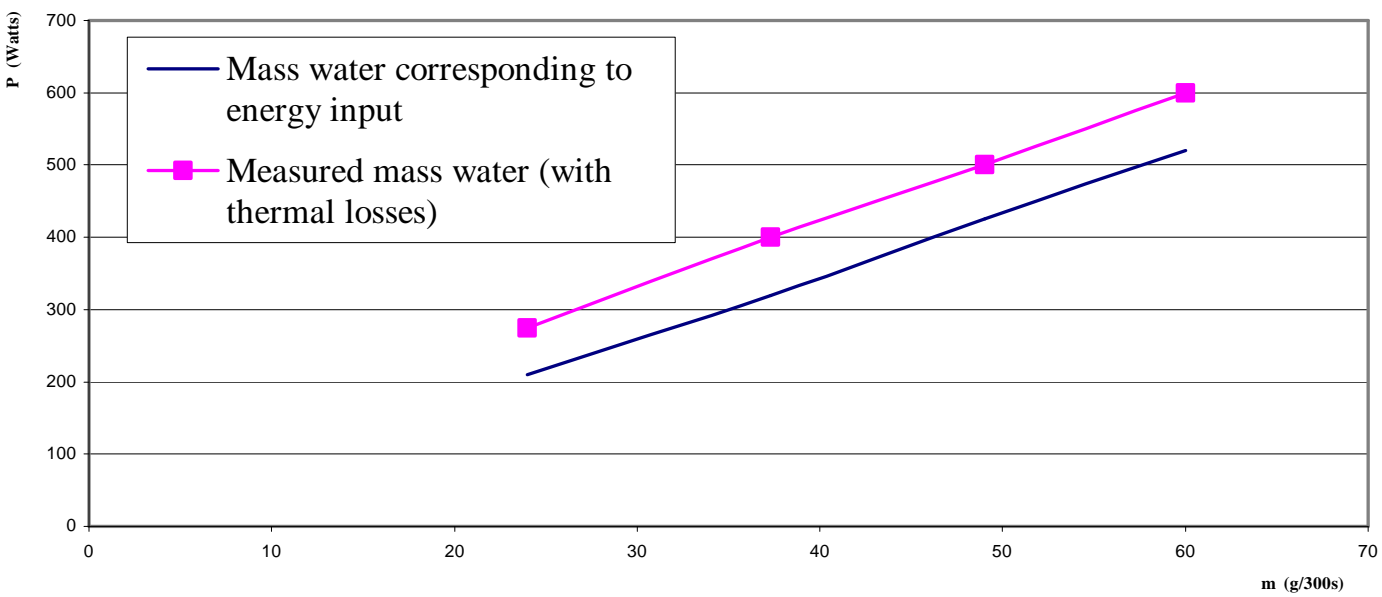

Figure 3. Device calibration using ohmic heating only.

\section{4-1 RESULTS}

We made a parametrical study on the influence of the voltage applied during glow discharge electrolysis. The temperature was brought to the boiling point $\left(100^{\circ} \mathrm{C}\right)$ with the auxiliary resistance heater. Then electrolysis was begun at 200 volts. This high voltage is needed to produce a suitable plasma around the cathode. Plasma generation is greatly enhanced by the high electrolyte temperature. The Joule heating effect during electrolysis immediately gives 
rise to an envelope of steam around the cathode, and a plasma, that is supposed to be hydrogen plasma according to Mizuno. The table shown below gives the value of the COP, which is the ratio of the energy of vaporization divided by the electrical energy supplied to the device (which of course includes energy consumed by the ohmic heater). It is very important to confirm that the device is thermally stable. The selected definitive values for the inlet energies are the values of the wattmeter, considered to be more reliable than those based on voltage and mean registered current.

Table 1. Five-minute test results

Voltage (volts)
COP (Energy out / energy in)

1.0 1.05
to

1.12

1.15

1.41
Number of tests

3

8

8

4

\section{Remarks:}

1- The calculation of the COP is straightforward. Let us take the 200 -volt data. The mass of evaporated water is $63 \mathrm{~g}$, with for $34 \mathrm{~g}$ due to auxiliary resistance heater. The mean current was 1.25 Amp., and the duration of the test is $300 \mathrm{~s}$ (5 minutes).

Energy out: $(63 \mathrm{~g}-34 \mathrm{~g}$ (auxiliary resistance) $\times 2594 \mathrm{j}=\underline{\mathbf{7 5 2 2 6}}$ joules

Energy in: $200 \mathrm{v} \times 1.25 \mathrm{~A} \times 300 \mathrm{~s}=\underline{\mathbf{7 5 0 0 0} \text { joules }}$

The COP is then:

2- $\quad$ The COP values bands are not correlated with the inaccuracy of the measurements. The results presented here were collected over several hours of operation of the cathode. The cathode is corroded at the very high temperatures of the experiment, so between experimental runs we push more of the cathode through the Pyrex shield, keeping the length of exposed cathode at about $15 \mathrm{~mm}$. That explains the variation bands.

3- Reproducibility is within a range of about $5 \%$. 


\section{4-2 DISCUSSION}

The very simple device used allowed us to highlight with reproducibility an abnormal excess heat, which increases with voltage.

We believe that we have confirmed abnormal excess energy for voltages higher than 200 volts with our present device. The data presented here for 350 volts correspond to input power exceeding 400 watts. The abnormal excess heat exceeds 120 watts without taking into account the gas formation or luminous radiation. This power is therefore definitely significant. We did not find any classical explanation for this thermal effect, and we are examining the possibility of less classical explanations.

\section{CONCLUSION}

The initial purpose of these experiments was to investigate the potential of a simple experimental device to confirm the results obtained by T. Mizuno. We think that this first step has been accomplished and that we can say that ratios between output and input energy (COP) of 1.3 and 1.4 have been reached with satisfactory reproducibility.

In a second step, we will try to explain this phenomenon in order to increase the COP.

In any case, the device presented in this paper is a very simple device which can be used to rapidly verify hypotheses without sophisticated means. For example, we have verified that this phenomenon does not seem related with the heavy water which is found at one part in 7,000 in natural water. We increased the concentration of heavy water by a factor of 100 in our electrolyte, i.e. to 1 part in 70 . We did not see any perceptible change in our results. 


\section{AKNOWLEDGEMENTS}

The authors want to express our deep appreciation to M. M. D. NOËL, J-L. NAUDIN and O. HORNER of "EDF Les Renardières" for fruitful discussions.

\section{REFERENCES}

Ref. 1. Experimental study of glow discharge in light water with Tungsten electrodes by D.Y. Chung, Y. Aoki, F. Senftle and T. Mizuno, ICCF11 conference in Marseille (Oct.31- Nov.5 / 2004).

T. Mizuno, T. Akimoto \& T. Ohmori. Confirmation of anomalous hydrogen generation by plasma electrolysis, in $4^{\text {th }}$ Meeting of Japan CF Research Society. 2003, Japan, Iwate University.

Ref. 2. Transmutation of metal to low energy in confined plasma in the water (electrochemical plasma cell) by D. Cirillo, A. Dattilo, V. Iorio, ICCF11 conference in Marseille (Oct.31 - Nov.5 / 2004). 